\title{
A Low-Power ASIC Design for Cell Search in the W-CDMA System
}

\author{
Chi-Fang Li, Student Member, IEEE, Yuan-Sun Chu, Wern-Ho Sheen, Member, IEEE, Fu-Chin Tian, and \\ Jan-Shin Ho, Student Member, IEEE
}

\begin{abstract}
This paper presents a low-power ASIC design for cell search in the wideband code-division multiple-access (W-CDMA) system. A low-complexity algorithm that is able to work satisfactorily under the effect of large frequency and clock errors is designed first. Then, a set of low-power measures are employed in the design of hardware architecture and circuits. Finally, through power analysis, critical blocks are identified and redesigned so as to further reduce the power consumption. The final design shows that the power is reduced by $51 \%$ from the original design of 133.6 $\mathrm{mW}$ to $65.49 \mathrm{~mW}$, and its core area is also reduced by $31.9 \%$ from $3.4 \times 3.4 \mathrm{~mm}^{2}$ to $2.8 \times 2.8 \mathrm{~mm}^{2}$. The design is implemented and verified in a 3.3-V $0.35-\mu \mathrm{m}$ CMOS technology with clock rate 15.36 MHz.
\end{abstract}

Index Terms-Cell search, clock error, frequency error, low-power design, W-CDMA.

\section{INTRODUCTION}

$\mathbf{I}$ N A CODE-DIVISION multiple-access (CDMA) cellular system, the procedure employed by a mobile station to search for the best cell site and to achieve code, time, and frequency synchronization with it is referred to as cell search. Fast cell search is particularly important for the wideband CDMA (W-CDMA) system because of the use of nonsynchronous base stations in the system [1], [2].

A three-stage search procedure has been designed in the W-CDMA specifications in order to facilitate fast cell search, including slot synchronization, joint frame synchronization and code-group identification, and scrambling-code detection [2]. Slot synchronization (stage 1) is achieved by detecting the primary synchronization channel $(\mathrm{PSCH})$. Joint frame synchronization and code-group identification (stage 2) is achieved by detecting the secondary synchronization channel (SSCH). And, after the code group is identified, the scrambling code can be determined easily by using the common pilot channel (CPICH) (stage 3).

A great deal of research has been contributed to the design of the cell search algorithms [1], [3]-[5]. In [1], a pipelined process was proposed to achieve faster cell search than the serial one at the cost of higher complexity. Partial symbol de-spreading with noncoherent combining was proposed in [1], [3] to overcome large frequency error due to the oscillator inaccuracy of

Manuscript received March 6, 2003; revised December 15, 2003. This work was supported by the Chip Implementation Center, National Science Council, under Grant NSC92-2218-E-194-008.

C.-F. Li, Y.-S. Chu, F.-C. Tian, and J.-S. Ho are with the Department of Electrical Engineering, National Chung-Cheng University, Chia-Yi 621, Taiwan, R.O.C. (e-mail: richard@vlsi.ee.ccu.edu.tw).

W.-H. Sheen is with the Department of Communication Engineering, National Chiao Tung University, Hsinchu 300, Taiwan, R.O.C. (e-mail whsheen@cm.nctu.edu.tw).

Digital Object Identifier 10.1109/JSSC.2004.826337 a mobile station. This imperfection also incurs sampling error (clock error) at the analog-to-digital converter (ADC). It was shown in [4] that the clock error may exceed over one timing period during the course of three-stage search and that will result in search failure. In [5], a search scheme with multiple "code time" candidates was proposed to reduce the search time in the clock-drifting environment.

In this paper, a low-power ASIC is designed for cell search in the W-CDMA system under the effect of large frequency and clock errors. A set of low-power design practices starting from the algorithm to hardware architecture and circuits is performed so as to reduce chip's power consumption. By using the power-efficient algorithm, architecture, and circuit designs, the power consumption of the design is reduced by $51 \%$. The design is implemented and verified in a $3.3-\mathrm{V} 0.35-\mu \mathrm{m}$ CMOS technology with clock rate $15.36 \mathrm{MHz}$.

The rest of this paper is organized as follows. Section II presents a low-complexity cell search algorithm under large frequency and clock errors. Section III describes low-power architecture and circuits, power analysis, and redesign of critical blocks. Section IV summarizes implementation and testing results. Finally, the paper is concluded in Section V.

\section{Low-COMPleXITy CELl SEARCh AlgorithM}

Three stages of cell search can be performed either in the serial or pipelined fashion [1]. In the pipelined search, all three stages are performed concurrently and that results in a faster search. In this paper, a low-complexity pipelined search algorithm will be adopted for the low-power ASIC design.

Different methods have been proposed to counteract the effects of frequency and clock errors on the cell search performance. Generally, two methods can be used to mitigate the effect of large frequency error. One is frequency offset compensation (FOC) and the other is partial symbol spreading (PSD). FOC has superior performance but needs multiple stage-1 detectors [1], [4]. To counteract the clock error, the simplest method is the random sampling per frame (RSPF) proposed in [4], which was shown to be able to work satisfactorily under $4 \mathrm{ppm}$ of clock error. For large clock error, the method of multiple timing candidates (MTC) could be employed, but multiple stage- 2 and stage- 3 detectors are needed [5]. Here, a simple method called sample-point reordering (SPR) is proposed to counteract the clock error for up to $10 \mathrm{ppm}$. The basic idea is as follows. First, the range of clock error is divided into "bins" with each bin denoting a presumed clock error. Then, within a bin, a controller is used to drop or stuff one sample point from or into incoming sampled sequence whenever the 


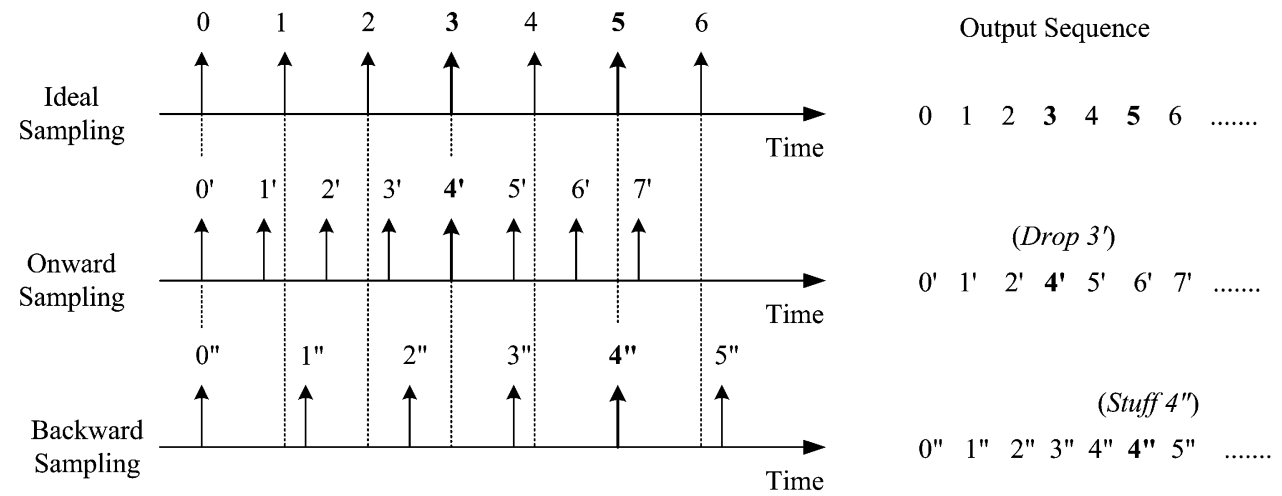

Fig. 1. Sample-point reordering.

TABLE I

COMPLEXITy of Different SEARChING Algorithms IN TERMS OF MOPS

\begin{tabular}{c|c|c|c|c|c|c|c|c|c|c}
\hline \hline & Default SPR & FOC & PSD & MTO & RSPF+FOC+SPR & RSPF+FOC+MTC & RSPF+PSD+SPR & RSPF+PSD+MTC \\
\hline \hline SPR & $3.84 S$ & - & - & - & - & $3.84 S\left(2+\mathrm{B}_{1}\right)$ & - & $3.84 S\left(2+\mathrm{B}_{1}\right)$ & - \\
\hline RSPF & $3.84 S$ & - & - & - & - & $3.84 S\left(2+\mathrm{B}_{1}\right)$ & $3.84 S\left(2+\mathrm{B}_{1}\right)$ & $3.84 S\left(2+\mathrm{B}_{1}\right)$ & $11.52 S$ \\
\hline FOC & 23.04 & - & - & - & - & $23.04\left(2+\mathrm{B}_{1}\right)$ & $23.04\left(2+\mathrm{B}_{1}\right)$ & - & - \\
\hline Stage 1 & 115.46 & $\times \mathrm{B}_{1}$ & $\times \mathrm{B}_{1}$ & 199.94 & - & $115.46 \mathrm{~B}_{1}$ & $115.46 \mathrm{~B}_{1}$ & $199.94 \mathrm{~B}_{1}$ & 199.94 \\
\hline Stage 2 & 13.72 & - & - & 14.02 & $\times \mathrm{L}_{2}$ & 13.72 & $13.72 \mathrm{~L}_{2}$ & 14.02 & $14.02 \mathrm{~L}_{2}$ \\
\hline Stage 3 & 123.36 & - & - & 124.8 & $\times \mathrm{L}_{3}$ & 123.36 & $123.36 \mathrm{~L}_{3}$ & 124.8 & $124.8 \mathrm{~L}_{3}$ \\
\hline Total & - & - & - & - & - & $\begin{array}{c}7.68 S\left(2+\mathrm{B}_{1}\right)+ \\
138.5 \mathrm{~B}_{1}+183.16\end{array}$ & $\begin{array}{c}3.84 S\left(2+\mathrm{B}_{1}\right)+ \\
138.5 \mathrm{~B}_{1}+13.72 \mathrm{~L}_{2} \\
+123.36 \mathrm{~L}_{3}+46.08\end{array}$ & $\begin{array}{c}7.68 S\left(2+\mathrm{B}_{1}\right)+ \\
199.94 \mathrm{~B}_{1}+138.82\end{array}$ & $\begin{array}{l}11.52 S+199.94+ \\
14.02 \mathrm{~L}_{2}+124.8 \mathrm{~L}_{3}\end{array}$ \\
\hline \hline
\end{tabular}

clock error is accumulated to one sample interval, as shown in Fig. 1.

Table I compares the complexity of different cell search algorithms in terms of million operations per second (MOPS) [6]. Among them, RSPF + PSD + SPR does not need any multiplier as used in the FOC function and has low complexity under the case of $\mathrm{B}_{1}=3, \mathrm{~L}_{2}=10, \mathrm{~L}_{3}=10$, where $\mathrm{B}_{1}$ is the number of stage 1 when using FOC and SPR, and $\mathrm{L}_{2}$ and $\mathrm{L}_{3}$ are the number of candidates associated with MTC for stages 2 and 3 , respectively. Fig. 2 shows the search time performance. The $\mathrm{RSPF}+\mathrm{PSD}+\mathrm{SPR}$ algorithm is able to reach 0.9 probability of search success in $600 \mathrm{~ms}$. It is good enough for practical applications and is adopted as the low-complexity algorithm for this low-power ASIC design.

\section{LOW-POWER HARDWARE DESIGN}

Fig. 3 illustrates the block diagram of cell search ASIC along with internal bitwidths [9]. The ASIC consists of four parts, including preprocessing, stage-1, stage-2, and stage-3 detectors. The preprocessing module includes RSPF and SPR. The sample-point reorder consists of a pair of tapped-delay lines along with multiplexers and a reordering controller. The initial selection of multiplexers is at the " 0 " position. Whenever the reordering controller accumulates sampling error up to one sampling interval, the selection of multiplexers is adjusted toward " $+/-$ " to drop or stuff one sampling point from or into incoming sampled sequence. Then, the RSPF selects one of the sampling points within one-chip duration as input data to the three stages and changes its selection randomly for every new frame.

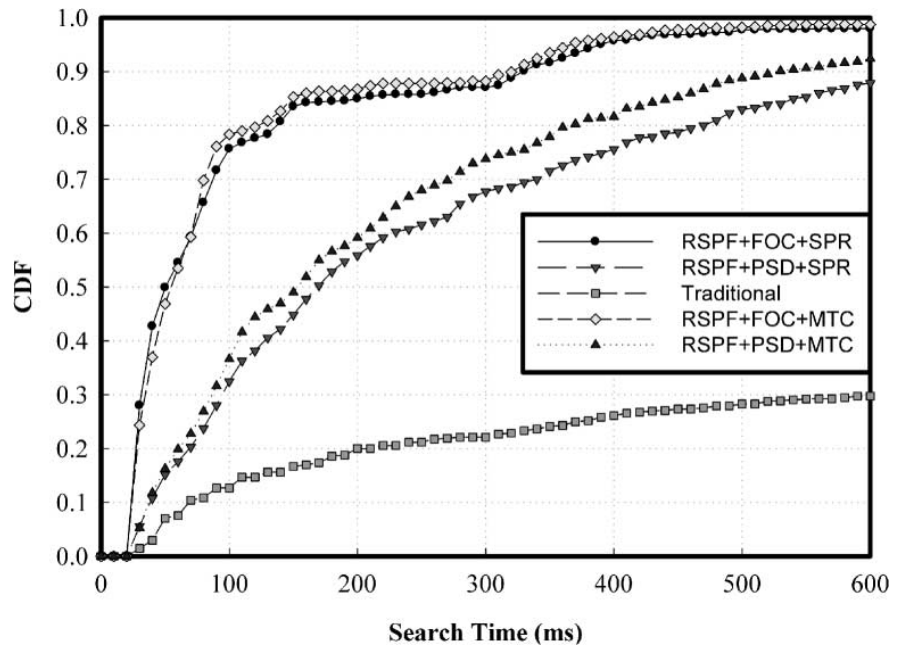

Fig. 2. Search performance of different algorithms. (In a frequency nonselective Rayleigh channel with frequency offset $=24 \mathrm{kHz}$, Doppler frequency $=185.2 \mathrm{~Hz}, \mathrm{~B}_{1}=3, \mathrm{~L}_{2}=10$, and $\mathrm{L}_{3}=10$ ).

In stage 1, the primary synchronization code (PSC) detector is designed as a hybrid de-spreader combining efficient Golay correlator (EGC) and hierarchical matched filter (MF) [7], [8]. As shown in Fig. 4, only 18 additions are needed to match PSC in four segments of 64-chip partial symbols. Similarly, a hybrid secondary synchronization code (SSC) detector is also designed by combining EGC and active correlator in stage 2 [9]. In stage 3, eight complex-valued active de-spreaders are employed to de-spread all scramble codes in the identified group.

After the first-phase design, a complete power analysis is performed through its post-layout simulations in a 3.3-V $0.35-\mu \mathrm{m}$ CMOS technology by the EPIC's Powermill tool. The driving 


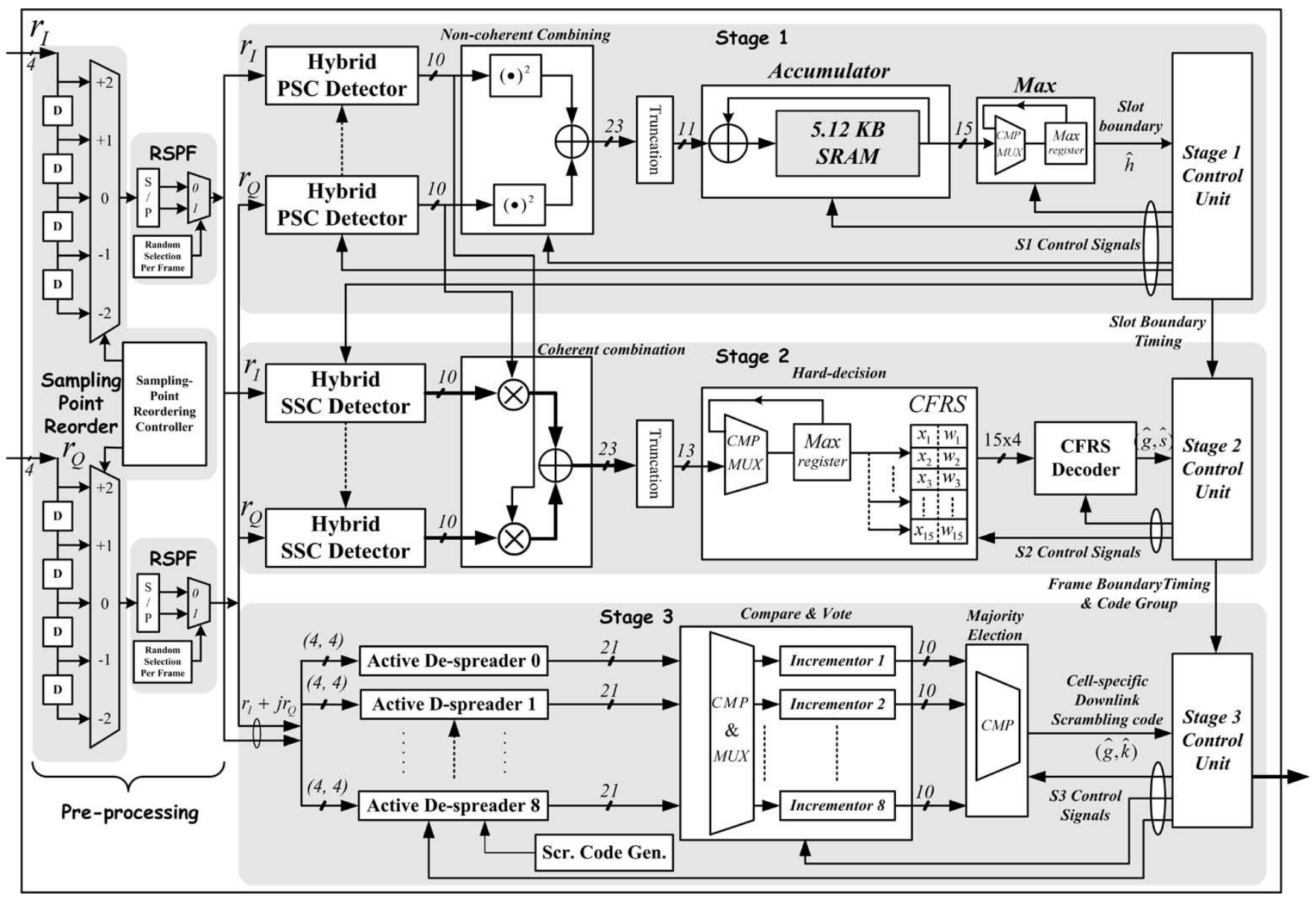

Fig. 3. ASIC block diagram.

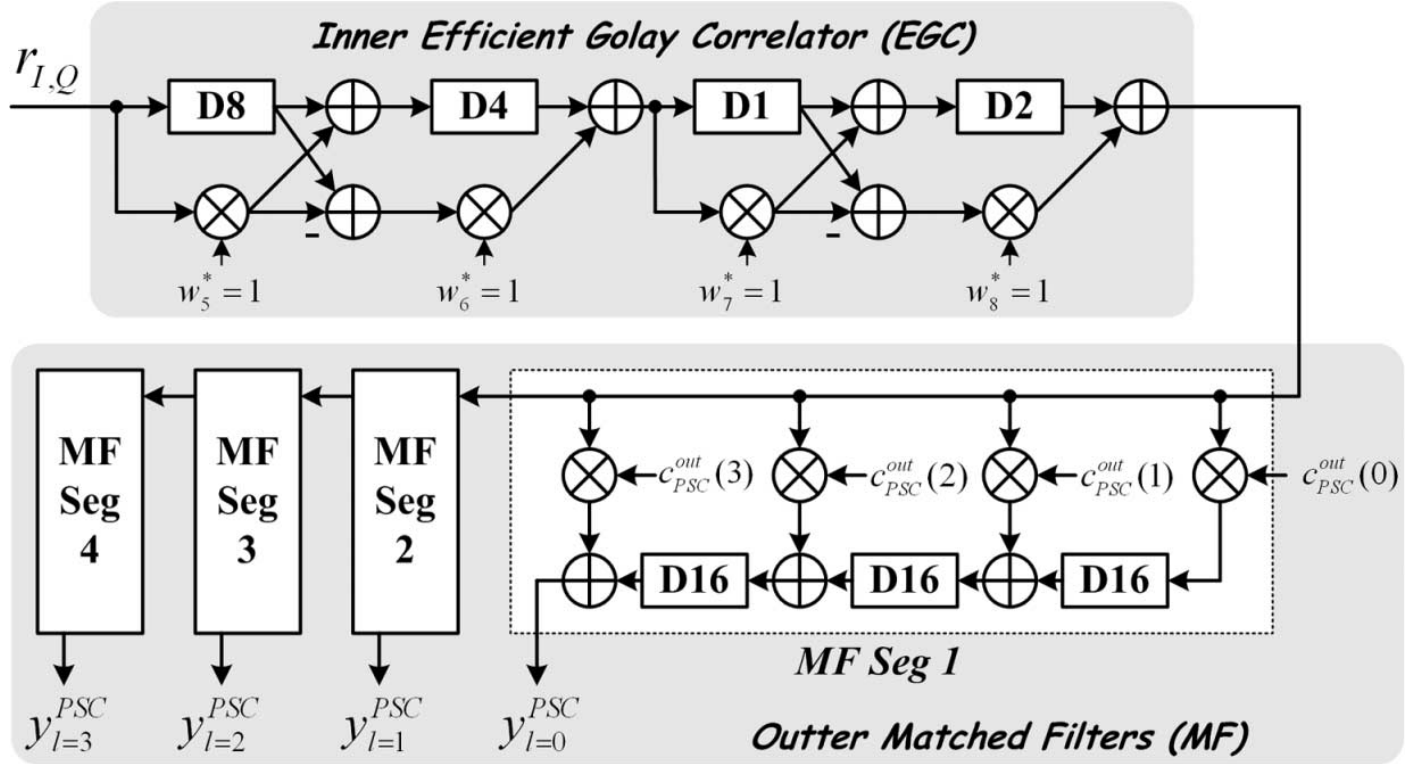

Fig. 4. Hybrid EGC-HMF detector for PSC. Di: Delay for $i$ clocks. It is implemented by using a shift register with $i$ fields.

vector is generated from the system simulation model in [4] and its length is over $600 \mathrm{~ms}$. Table II tabulates the details of power consumption and layout areas of all blocks.

As shown in the table, de-spreaders and (non)coherent combiners are the power-critical bocks in cell search processing. In de-spreaders, many shift registers are used in EGCs and MFs of PSC and SSC detectors. These shift registers are used as delay elements only, but all fields of shift registers change their values per clock cycle. This value (level) changing in CMOS circuits results in unwanted power dissipation. To overcome this defi- 


\section{In Theory:}
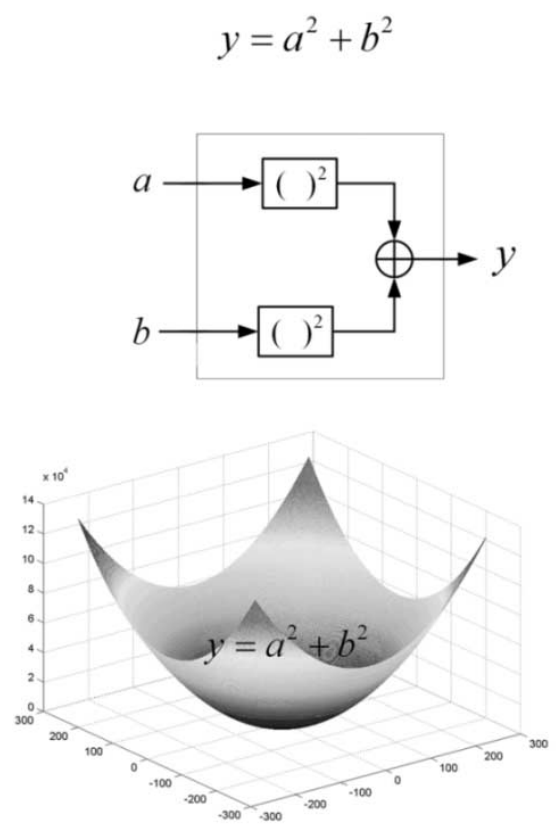

Fig. 5. New approximation of magnitude calculations.

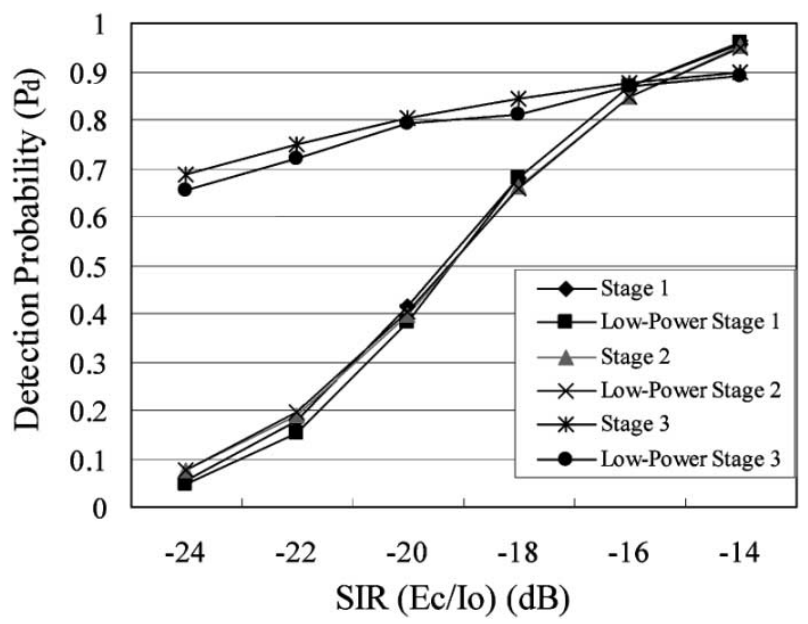

Fig. 6. Simulation results using the new magnitude approximation. (In a frequency nonselective Rayleigh channel with frequency offset $=0 \mathrm{kHz}$, Doppler frequency $=200 \mathrm{~Hz}$ ).

ciency, a pointer-based FIFO buffer is used to replace shift registers [10].

In (non)coherent combiners, main operations are multiplications or squares used to calculate the true magnitude of signals. In cell search, however, magnitude values of signals are just used to compare with each other for finding the largest one. Therefore, a new low-complexity approximation is proposed to reduce the power consumption. That is, $y=a^{2}+b^{2}$ is approximated by

$$
\begin{aligned}
& y=\alpha|a|+\beta|b|, \\
& \alpha=2^{d} \text { if } 2^{d-1} \leq|a|<2^{d}, \\
& \beta=2^{d} \text { if } 2^{d-1} \leq|b|<2^{d}
\end{aligned}
$$

where $\alpha$ and $\beta$ are of the form $2^{d}$ and therefore, only shift operations are needed. Fig. 5 shows the curve plane of $y=\alpha|a|+\beta|b|$

\section{New Proposal:}

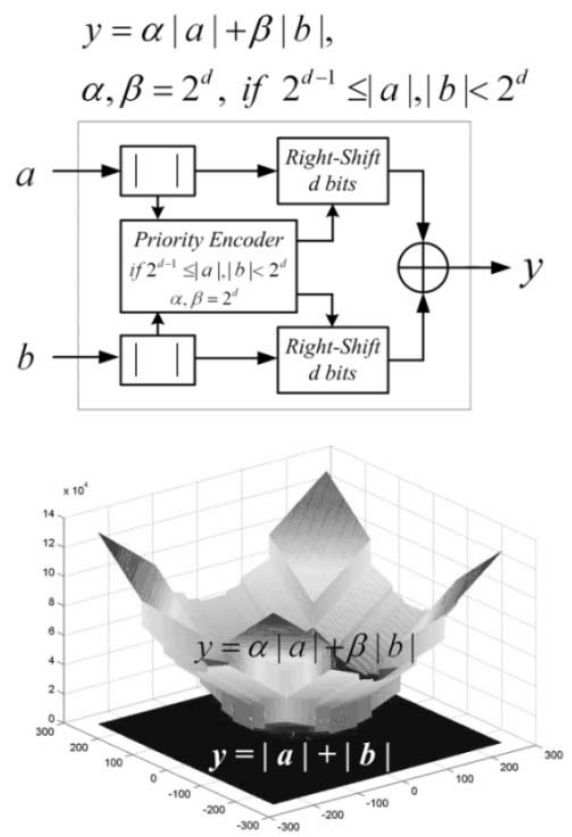

\begin{tabular}{|c|c|c|c|c|}
\hline & Blocks & $\begin{array}{c}\text { Gate } \\
\text { Count }\end{array}$ & $\begin{array}{c}\text { Area } \\
\left(\mathrm{mm}^{2}\right)\end{array}$ & $\begin{array}{l}\text { Power } \\
(\mathrm{mW})\end{array}$ \\
\hline & Sample reorder & 104 & 0.0441 & 0.54 \\
\hline & RSPF & 85 & 0.0361 & 0.44 \\
\hline & PSC detector $\times 2$ & 2,924 & 2.88 & 30.2 \\
\hline & Non-coherent combiner & 8,525 & 2.89 & 62.7 \\
\hline S1 & Accumulator & 94 & 0.0144 & 0.01 \\
\hline & Maximum selector & 197 & 0.0289 & 0.11 \\
\hline & Stage- 1 controller & 262 & 0.0529 & 0.17 \\
\hline & Stage 1 & 13,338 & 5.8322 & 93.19 \\
\hline & SSC detector $\times 2$ & 3,458 & 1.8 & 4.85 \\
\hline & Coherent combiner & 4,149 & 0.8464 & 1.98 \\
\hline S2 & CFRS symbol detector & 415 & 0.087 & 0.33 \\
\hline & CFRS decoder & 1,481 & 0.36 & 1.32 \\
\hline & Stage- 2 controller & 290 & 0.0529 & 0.19 \\
\hline & Stage 2 & 8,091 & 2.7495 & 8.67 \\
\hline & Active De-spreader $\times 8$ & 18,328 & 2.8224 & 27.2 \\
\hline & Scrambling-code gen. & 1,007 & 0.2352 & 2.2 \\
\hline $\mathrm{S} 3$ & Voter & 349 & 0.0675 & 0.13 \\
\hline & Majority selector & 378 & 0.1155 & 0.47 \\
\hline & Stage- 3 controller & 641 & 0.0841 & 0.76 \\
\hline & Stage 3 & 21,140 & 3.3635 & 30.76 \\
\hline & Whole chip & 42,547 & $3.4 \times 3.41$ & 133.6 \\
\hline
\end{tabular}

TABLE II

POWER CONSUMPTIONS AND CORE AREAS OF CELL SEARCH COMPONENTS

which is apparently much closer to that of $y=a^{2}+b^{2}$ than other approximations, such as $y=|a|+|b|$ and $y=|a|+|b|+$ $\max (|a|,|b|)$. Fig. 6 is the simulation result by using this approximation. It is clear that the approximation induces almost no loss on search performance.

After applying the pointer-based FIFO buffer and the new magnitude calculator into the cell search ASIC, the power reduction is $51 \%$ from the original design of $133.6 \mathrm{~mW}$ to 65.49 $\mathrm{mW}$. In addition, the chip area is also reduced $31.9 \%$ from $3.4 \times$ $3.4 \mathrm{~mm}^{2}$ to $2.8 \times 2.8 \mathrm{~mm}^{2}$. Fig. 7 shows power reduction of redesigned blocks. 


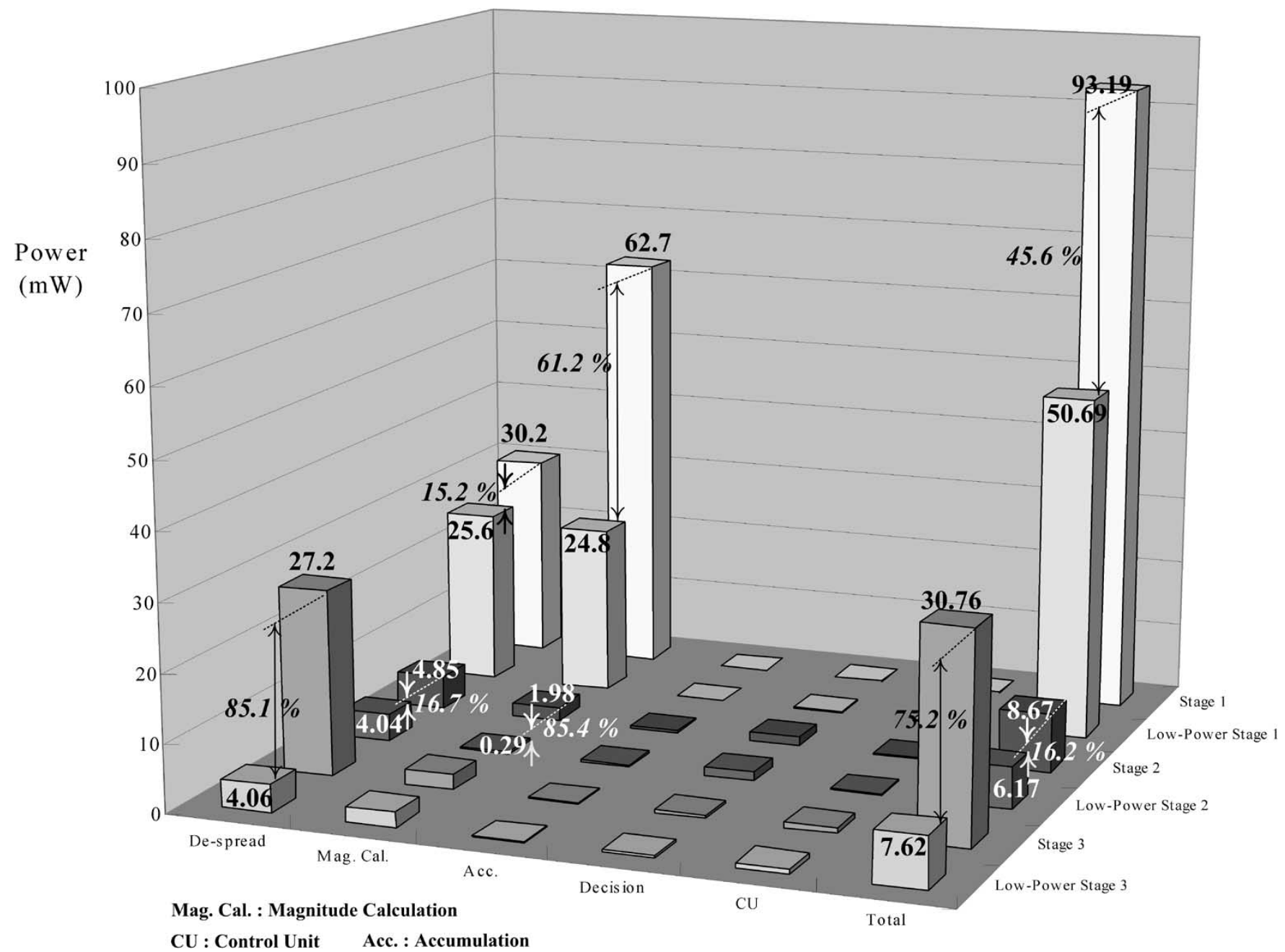

Fig. 7. Power consumption of different blocks.

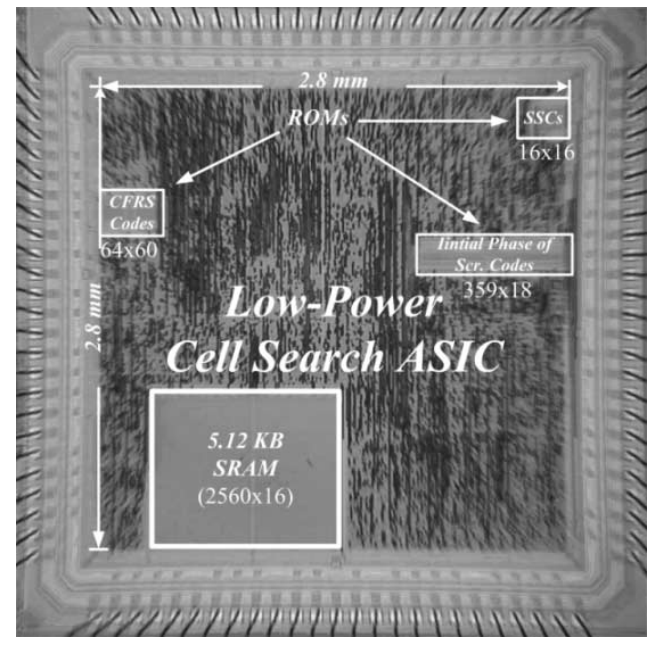

Fig. 8. Chip photograph.

\section{IMPLEMENTATION RESULTS}

The low-power cell search ASIC is implemented into a real chip through a top-down cell-based ASIC design approach
TABLE III

CHIP PROFILE

\begin{tabular}{c|c}
\hline \hline \multicolumn{2}{c}{ Low-Power Cell Search ASIC } \\
\hline \hline Technology & $3.3-\mathrm{V} 0.35-\mu \mathrm{m}$ CMOS 1P4M process \\
\hline Transistor Count & 346,142 \\
\hline Core Area & $2.8 \times 2.8 \mathrm{~mm}^{2}$ \\
\hline Chip Size & $4.3 \times 4.3 \mathrm{~mm}^{2}$ \\
\hline Package & $100 \mathrm{CQFP}$ \\
\hline Power Dissipation & $65.83 \mathrm{~mW} \mathrm{@} 15.36 \mathrm{MHz}$ \\
\hline Max. Speed & $50 \mathrm{MHz}$ \\
\hline
\end{tabular}

in a 3.3-V $0.35-\mu \mathrm{m}$ CMOS technology. Fig. 8 is the chip photograph. Three ROMs are used to store the comma-free Reed-Solomon (CFRS) codebook $(64 \times 60), 16$ SSC outer codes $(16 \times 16)$, and 359 initial phases of scrambling codes $(359 \times 18)$. A 5.12-kB SRAM is used to accumulate correlation results of 15 slots for 2,560 slot-boundary hypotheses in stage 1. The chip profile is summarized in Table III. The chip consumes $65.83 \mathrm{~mW}$ at $15.36 \mathrm{MHz}$. It is a little more than the simulation result. 


\section{CONCLUSION}

A low-power cell search ASIC for the W-CDMA system has been designed and implemented. First, a low-complexity search algorithm, which combines sample-point reordering, random sampling per frame, and partial symbol de-spreading is devised to counteract the effects of large frequency and sampling errors. The algorithm is then implemented to its layout with careful consideration for power consumption. Furthermore, the power consumption of whole chip is analyzed, and critical blocks such as those of de-spreading and magnitude calculations are redesigned using pointer-based FIFO buffers and new weighted magnitude calculators. The power and area reductions of the chip are $51 \%$ and $31.9 \%$, respectively. The low-power cell search ASIC consumes $65.49 \mathrm{~mW}$ at $15.36 \mathrm{MHz}$.

\section{REFERENCES}

[1] Y.-P. E. Wang and T. Ottosson, "Cell search in W-CDMA," IEEE J. Select. Areas Commun., vol. 18, pp. 1470-1482, Aug. 2002.
[2] "Spreading and Modulation (FDD)," 3rd Generation Partnership Project, 3GPP Tech. Spec. TS 25.213, V3.7.0, Dec. 2001.

[3] K.-M. Lee and J.-Y. Chun, "An initial cell search scheme robust to frequency error in W-CDMA system," in Proc. IEEE Int. Symp. Personal, Indoor and Mobile Radio Communications, vol. 2, Sept. 2000, pp. 1400-1404.

[4] W.-H. Sheen and J.-S. Ho, "Cell search for 3GPP W-CDMA/FDD with chip clock shift and non-ideal sampling," in Proc. IEEE Vehicular Technology Conf., vol. 4, Oct. 2001, pp. 2369-2374.

[5] M. Kiessling and S. A. Mujtaba, "Performance enhancements to the UMTS (W-CDMA) initial cell search algorithm," in Proc. IEEE Int. Conf. Communication, vol. 1, 2002, pp. 590-594.

[6] C.-F. Li, W.-H. Sheen, and Y.-S. Chu, "An integrated multi-scheme cell search platform for W-CDMA applications," in Proc. IEEE Int. Symp. Personal, Indoor and Mobile Radio Communications, vol. 1, Sept. 2003, pp. 1400-1404.

[7] B. M. Popovic, "Efficient Golay correlator," IEE Electron. Lett., vol. 33, no. 17, pp. 1427-1428, Aug. 1999.

[8] "A new hierarchical correlation sequence with good properties in presence of a frequency error," Siemens, 3GPP TSG RAN W1 Tdoc 99/146.

[9] C.-F. Li, W.-H. Sheen, and Y.-S. Chu, "ASIC design for cell search in 3GPP W-CDMA/FDD," in Proc. IEEE Vehicular Technology Conf., vol. 3, Oct. 2001, pp. 1383-1387.

[10] E. K. Tsern and T. H. Meng, "A low power video-rate pyramid VQ decoder,” IEEE J. Solid-State Circuits, vol. 31, pp. 1789-1794, Nov. 1996. 